Conclusion CRP has good specificity ( $96 \%$ at $1 \mathrm{mg} / \mathrm{L}$ ) for CA in preterm infants. Higher initial CRP levels in infants correlate with severity of histological CA.

\section{PO-0564 TURN-AROUND-TIMES FOR PATHOGEN IDENTIFICATION AND ANTIBIOTIC SUSCEPTIBILITY TESTING IN INFANTS WITH EARLY-ONSET BACTERIAL SEPSIS}

S Sarkar, SS Sarkar. Neonatal-Perinatal Medicine, University of Michigan Health System, Ann Arbor, USA

\subsection{6/archdischild-2014-307384.1206}

We characterised the turn-around-times for pathogen identification with antibiotic susceptibility, and outcomes in newborn infants with early-onset bacterial sepsis (EOS).

Methods Eighty infants with EOS were retrospectively reviewed. EOS was defined by isolation of a pathogen from blood culture drawn within $72 \mathrm{~h}$ of birth and antibiotic treatment for $\geq 5$ days.

Results Thirty-seven of the 80 infants were deemed to have true EOS, and 43 were deemed contaminants. The organisms grown in true EOS cases were: E. Coli in 16, Group B Streptococcus in 10, Alpha hemolytic Streptococci in 6, and others in 5 .

The median (25\%-75\% IQR) time noted from blood culture positivity to identification of the organisms with susceptibility testing was almost 4 times longer compared to the time from collection of blood culture specimens to blood culture positivity (79 h, IQR $52 \mathrm{~h}-101 \mathrm{~h}$, versus $19 \mathrm{~h}$, IQR $16 \mathrm{~h}-21 \mathrm{~h}, \mathrm{p}<$ 0.0001) in true cases of EOS. The contaminants took longer to identify compared to true cases $(\mathrm{p}<0.05)$.

Four infants died of gram negative sepsis. Two of these infants with ampicillin resistant E. Coli died from delayed implementation of appropriate organism-specific antibiotic treatment as the susceptibility results took too long to become available.

Conclusions Definitive identification of the pathogen with the currently used laboratory methods take too long affecting outcome of infants with EOS. Empiric antibiotics were continued too long unnecessarily because of delayed identification of the contaminants. Rapid identification of an organism to a species level utilising newer technologies needs to be developed.

\section{PO-0565 FOUR YEARS COHORT OF LATE PRETERM INFANTS FROM A TERCIARY SPANISH HOSPITAL: RISK FACTORS FOR RESPIRATORY SYNCITIAL VIRUS INFECTIONS}

L Serrano López, L Zamorano Bonilla, MV Jimenez Cabanillas, E Martin Alvarez, M Peña Caballero, JA Hurtado Suazo. Neonatology, University Hospital "Virgen de Las Nieves", Granada, Spain

\subsection{6/archdischild-2014-307384.1207}

Background and aims Respiratory Syncitial Virus (RSV) causes respiratory infections that may be severe, especially in one of the highest risk populations: Premature infants. Palivizumab has proven safe and efficacious in reducing hospitalisation rate for RSV induced bronchiolitis in preterm neonates.

This study aimed to evaluate in a Spanish cohort of late preterm infants the incidence and risk factors of hospitalisation for RSV bronchiolitis. Update the indication of RSV prophylaxis.
Methods Descriptive study. A cohort of late preterm infants born in a Spanish third level hospital (2010-2014) was enrolled. Medical records were reviewed. Risk factors for RSV infections were reviewed. Recommendations for inmunoprophylaxis issued by the Spanish Society of Neonatology 2010 were followed.

Results 887 late preterm infants were enrolled. 4,1\% were hospitalised for RSV bronchiolitis, median age was 5 months old. According to the gestational age: 16\% were 34 weekers (one RSV prophylaxis), 45\% 35 weekers (three RSV prophylaxis), and 38\% 36 weekers (one RSV prophylaxis).

The risk factors for RSV hospitalisation: $56 \%$ were born in RSV season, $48 \%$ had school age siblings, 54\% were male gender. Anyone was exposed to passive cigarette smoke.

$16 \%$ were admitted to ICU. All the hospitalised infants required oxygen at any time. No deaths were reported.

Conclusions Hospitalisation rate for RSV bronchiolitis in late preterm infants of our cohort was higher than the estimated one in overall population. RSV prophylaxis was not routinely scheduled to late preterm infants according to the Guidelines issued by the Spanish Society of Neonatology. Its risk scoring tool for prophylaxis can be used to identify infants at higher risk.

\section{P0-0566 PLATELETS AS THE OPSONINS THAT PROMOTES INGESTION OF MICROBES DURING NEONATAL SEPSIS}

${ }^{1} \mathrm{M}$ Sherman, ${ }^{1} \mathrm{~L}$ Wahidi, ${ }^{2} \mathrm{~J}$ Sherman. 'Child Health, University of Missouri, Columbia, USA; ${ }^{2}$ Sinclair School of Nursing, University of Missouri, Columbia, USA

\subsection{6/archdischild-2014-307384.1208}

Background and aims Complement and IgG are humoral opsonins. We theorised platelets might be opsonins in neonates. We proposed that persistent neonatal bloodstream infections and thrombocytopenia might provide proof of the concept if there was high rather than low mean platelet volumes [MPVs] during infections (i.e., platelets consumed during phagocytosis).

Methods From 2008 to 2013, all neonates >3 days of age and that had positive blood cultures underwent a record review. Infants were included if they had $\geq 2$ positive blood cultures and had platelet counts $<10^{5}$ per $\mathrm{mm}^{3}$. Exclusion criteria were necrotizing enterocolitis, coagulopathy, organ or catheter-related thrombosis or endocarditis.

Results Among 77 positive blood cultures, two methicillin-resistant Staphylococcus aureus [MRSA] and two Candida bloodstream infections persisted and had thrombocytopenia. The four infants had initial elevated MPVs that declined to normal only with the resolution of infection. Blood smears had no aggregates of platelet, microbes and phagocytes. One MRSA and two Candida infections with associated thrombocytopenia occurred in extremely preterm infants; they had no elevation in MPVs and expired quickly. A review of all 77 infants with late-onset sepsis revealed the infecting microbe and extreme prematurity modulated the kinetics of MPVs during infection.

Conclusions Two pathogens that likely resisted opsonization with complement and IgG were associated with continuing neonatal sepsis and thrombocytopenia. High MPVs suggests defective platelet production was not responsible for thrombocytopenia, but macrophages and neutrophils likely removed platelet-microbe-aggregates from the blood. These findings offer indirect proof that platelets may act as opsonins during neonatal phagocytosis. 